\title{
Investigation of Comorbidity, Trauma History, and Osteoporotic Fractures in the Postmenopausal Population: A Nationwide, Observational, and Cross-Sectional Study of Korean Orthopedic Outpatient Clinics
}

\author{
Minjoon Cho ${ }^{1}$, Seoung-Hwan Moon ${ }^{2}$, Ji-Ho Lee ${ }^{1,3}$, Jae Hyup Lee ${ }^{1,3}$ \\ ${ }^{1}$ Department of Orthopedic Surgery, SMG-SNU Boramae Medical Center, Seoul, Korea \\ ${ }^{2}$ Department of Orthopaedic Surgery, Yonsei University College of Medicine, Seoul, Korea \\ ${ }^{3}$ Department of Orthopedic Surgery, Seoul National University College of Medicine, Seoul, Korea
}

\begin{abstract}
Study Design: A nationwide, observational, and cross-sectional study targeting postmenopausal patients from 62 orthopedic outpatient clinics in Korea between October 2010 and February 2011.

Purpose: This study was carried out to investigate comorbidity, trauma history, and the status of osteoporotic fracture treatment in Korean postmenopausal women.

Overview of Literature: There has been little reports on the comorbidity, family history, trauma history, and treatment status of osteoporotic fractures in patients visiting the orthopedic outpatient clinics in Korea.

Methods: A total of 1,255 postmenopausal women between the ages of 50 and 80 years were enrolled in the study and the population distribution was reflected by region. Comorbidity, familial history of osteoporosis, familial history of osteoporotic fracture, history of falls, and status of osteoporotic fracture management were evaluated using an interview and questionnaire. The relationship between family history of osteoporosis and bone mineral density was analyzed.

Results: A number of patients (23\%) had a family history of osteoporosis and $16.5 \%$ had a family history of fractures. Most (64.7\%) of the patients had one or more comorbidities, including $58.8 \%$ exhibiting a chronic disease and $16.4 \%$ suffering from diseases that restrict exercise or walking. The results of the questionnaire indicated that $21.8 \%$ of these fracture patients had experienced a fracture previously and that the most common type of fracture was that of the spine. Lumbar spine bone mineral density was found to be lower in the presence of family history of osteoporosis.

Conclusions: Postmenopausal women are liable to have osteoporotic fractures due to the high prevalence of osteoporosis, a history of falling, and the comorbidity with diseases that restrict ambulation. A better understanding of postmenopausal women in the orthopedic outpatient settings is important to the management of osteoporotic fractures.
\end{abstract}

Keywords: Osteoporosis; Postmenopausal; Spinal fractures; Comorbidity; Trauma

Received Dec 8, 2018; Revised Dec 17, 2018; Accepted Jan 1, 2019

Corresponding author: Jae Hyup Lee

Department of Orthopaedic Surgery, SMG-SNU Boramae Medical Center, Seoul National University College of Medicine, 20 Boramaero 5-gil, Dongjak-gu, Seoul 07061, Korea

Tel: +82-2-870-2314, Fax: +82-2-870-3863, E-mail: spinelee@snu.ac.kr 


\section{Introduction}

Estrogen depression makes postmenopausal women more vulnerable to osteoporosis due to decreased osteoblast function. It is well known that increasing age is associated with decreasing bone mineral density (BMD) in postmenopausal women. According to a study published in 2017 [1], the average life expectancy of South Korean women is likely to exceed 90 years in 2030 and, in these women, complications such as osteoporosis and fractures will increase rapidly. Therefore, the clinical significance of osteoporosis and osteoporotic fractures in Korean postmenopausal women is expected to escalate further. As orthopedic surgeons are the first medical practitioners to come into contact with fracture patients, epidemiologic information gathered from orthopedic outpatient clinic patients is useful to them. However, orthopedic surgeons are often not familiar with the data even though postmenopausal women as a population have many comorbidities and risk factors for osteoporotic fractures. As there have been no previous reports measuring these parameters to date, the purpose of this study was to investigate comorbidity, family and trauma history, and status of osteoporotic fracture treatment in postmenopausal women who have visited orthopedic outpatient clinics in Korea.

\section{Materials and Methods}

\section{Study design}

This was a nationwide, observational, and cross-sectional study targeting postmenopausal patients in 62 orthopedic outpatient clinics between October 2010 and February 2011 in Korea. The methods are as described previously $[2,3]$. To compensate for regional differences between areas, data provided by the National Statistical Office of Korea were used to identify the distribution of women over the age of 50. The same proportion of patients was recruited from each of four regions, namely the Seoul Metropolitan, Central, Youngnam, and Honam areas. A total of 1,281 patients was enrolled in this study.

\section{Inclusion criteria}

Among women 50 years of age or older who visited orthopedic clinics, patients who had undergone menopause, as confirmed by a physician, were included. Postmenopausal patients were defined as women for whom 12 months had passed since their last menstrual period at the time of enrollment $[2,3]$.

\section{Exclusion criteria}

We excluded premenopausal women, subjects $\geq 80$ years of age, women who had undergone a high-energy trauma such as a traffic accident within the past 3 months, subjects who had suffered non-vertebral fractures in the 6 months prior to the study registration, and people who did not have the cognitive ability to be involved in the research (i.e., the skill to understand the contents of the survey) based on the discretion of the investigator $[2,3]$.

\section{Demographic information and medical history}

Patients were evaluated for age, height, weight, years following menopause, family history of osteoporosis in the mother and siblings, family history of osteoporotic fracture in the mother and siblings, living environment (residential area, facilitated residence, family residence, etc.), type of insurance (medical insurance or medical care), and medical record. In addition, we identified comorbidities such as hypertension, diabetes, hypercholesterolemia, osteoporosis, rheumatoid arthritis, hyperthyroidism, hyperparathyroidism, degenerative knee osteoarthritis, foot disease, spinal disease with claudication, and Parkinson's disease. Furthermore, details about any history of falls and traumas experienced within the year prior to the study period were noted. If there was a history of fracture, we determined whether the fracture occurred before or after the onset of menopause, the site and cause of the fracture, and the treatment received $[2,3]$.

\section{Bone mineral density}

The BMD of patients was measured at points in the lumbar spine, femoral neck, and total hip joint using dual energy X-ray absorptiometry. If data measured within 3 months prior to the study were available, they were used. Measurements were carried out according to the manufacturer's instructions for each measuring instrument. BMD data were statistically analyzed for correlation with a family history of osteoporosis, exercise- and movementlimiting diseases, and a history of steroid treatment $[2,3]$. 
Table 1. Family history of osteoporosis and fracture

\begin{tabular}{lccc} 
Item & No. of patients & Yes & No \\
Family history of osteoporosis $^{\text {a) }}$ & 888 & $205(23.1)$ & 683 (76.9) \\
Family history of fracture $^{\text {b) }}$ & 1,086 & $179(16.5)$ & 907 (83.5) \\
\hline
\end{tabular}

Values are presented as number (\%).

a)"Do not know" or no response (n=367). ${ }^{\text {b) }}$ Do not know" or no response (n=169).

\section{Ethics statement}

The study was conducted in accordance with the Declaration of Helsinki and approved by the institutional review board (IRB approval no., 06-2010-131) of Seoul Metropolitan Government-Seoul National University Boramae Medical Center. All patients provided written informed consent $[2,3]$.

\section{Results}

This study is a follow-up to research conducted in 2014 [2]. A total of 1,255 patients were included in this study. The mean age of the patients was $63.2 \pm 7.7$ years, the age at the time of menopause was $48.8 \pm 5.2$ years, and the mean postmenopausal period was $14.5 \pm 9.0$ years. Among the subjects, 209 (16.8\%) lived alone and 1,021 (82.3\%) lived with their families. A total of 1,083 (87.3\%) resided in a city or county, and $158(12.7 \%)$ resided in a town or township. According to the details collected about medical insurance, 1,155 (95.0\%) of the patients were enrolled as national health insurance subscribers and 59 (4.8\%) were receiving medical care.

\section{Family history}

Of the postmenopausal women who visited the orthopedic outpatient clinic, $23 \%$ had a family history of osteoporosis and $16.5 \%$ had a family history of fractures (Table 1). The response rates to questions about a family history of osteoporosis and family history of fractures were $70.7 \%$ and $86.5 \%$, respectively. Patients tended to remember a family history of fracture more than a family history of osteoporosis.

\section{Comorbidity}

The most common comorbidities among postmenopausal women who visited orthopedic outpatient clinics were
Table 2. Accompanying disease and history of steroid therapy $(\mathrm{N}=1,255)$

\begin{tabular}{|c|c|}
\hline Accompanying disease & No. $(\%)$ \\
\hline Hypertension & $491(39.1)$ \\
\hline Diabetes & $169(13.5)$ \\
\hline Hyperlipidemia & $154(12.3)$ \\
\hline Osteoporosis & $196(15.6)$ \\
\hline Rheumatoid arthritis & $23(1.8)$ \\
\hline Hyperthyroidism & $22(1.8)$ \\
\hline Hyperparathyroidism & $1(0.1)$ \\
\hline Severe knee arthritis & $140(11.2)$ \\
\hline Spinal disease with lag & $84(6.7)$ \\
\hline Foot disease & $20(1.6)$ \\
\hline Parkinson's disease & $5(0.4)$ \\
\hline Other & $123(9.8)$ \\
\hline At least one of the above diseases & $812(64.7)$ \\
\hline People with chronic disease ${ }^{a}$ & $738(58.8)$ \\
\hline Disease limiting exercise or walking ${ }^{\mathrm{b}}$ & $206(16.4)$ \\
\hline Steroid treatment for more than 3 months & $19(1.5)$ \\
\hline \multicolumn{2}{|c|}{$\begin{array}{l}\text { alChronic diseases: hypertension, diabetes, hyperlipidemia, osteoporo- } \\
\text { sis, rheumatoid arthritis, hyperthyroidism, hyperparathyroidism, lumbar } \\
\text { spinal diseases, and Parkinson's disease (excluding other diseases). } \\
\text { blDisease that restricts exercise or walking: severe knee osteoarthritis, } \\
\text { spine disease with claudication, foot disease, and Parkinson's disease } \\
\text { (excluding other diseases). }\end{array}$} \\
\hline
\end{tabular}

hypertension (49.1\%), osteoporosis (15.6\%), diabetes (13.5\%), hyperlipidemia (12.3\%), and arthritis of the knee (11.2\%). Table 2 summarizes the prevalence of other chronic illnesses or diseases. Most patients (64.7\%) had one or more comorbidities. The percentage of patients with chronic diseases such as hypertension, diabetes, hyperlipidemia, osteoporosis, rheumatoid arthritis, hyperthyroidism, hyperparathyroidism, spinal diseases with claudication, and Parkinson's disease was 58.8\%. In addition, the proportion of postmenopausal women with diseases that limited exercise or walking ability, such as severe knee osteoarthritis, spine disease with claudica- 
tion, foot disease, and Parkinson's disease was $16.4 \%$. The prevalence of rheumatoid arthritis, hyperparathyroidism, and a history of steroid injection lasting over 3 months were $1.8 \%, 0.1 \%$, and $1.5 \%$, respectively. All of these are possible causes of secondary osteoporosis.

\section{Causes and treatment of fractures with respect to fracture sites}

A number of patients $(n=274,21.8 \%)$ had experienced previous fractures at the same location. These included $8.3 \%$ of the patients who had spinal fractures; $5.7 \%$ who had wrist fractures; $2.2 \%$ who had hip fractures; and $1.0 \%$ who had knee fractures (Table 3). A total of $7.3 \%$ had experienced other fractures. The highest proportion of fractures were caused by falls (61.9\%), then external impact (11.6\%), and daily activities (11.2\%).

Most patients (81.5\%) with hip fractures underwent surgical treatment while patients with wrist fractures, vertebral fracture, and knee fracture were treated surgically

Table 3. Fracture site and cause of fracture $(N=1,255)$

\begin{tabular}{|c|c|c|c|c|c|c|}
\hline \multirow{2}{*}{ Fracture site } & \multirow{2}{*}{ Total } & \multirow{2}{*}{$\begin{array}{c}\text { No. of } \\
\text { occurrences }\end{array}$} & \multicolumn{4}{|c|}{ Cause of fracture } \\
\hline & & & Do not know & Fall & External impact & Daily activity \\
\hline Hip & $27(2.2)$ & 27 & $3(11.1)$ & $19(70.4)$ & $3(11.1)$ & $2(7.4)$ \\
\hline Knee & $12(1.0)$ & 12 & $2(16.7)$ & $6(50.0)$ & $2(16.7)$ & $2(16.7)$ \\
\hline Spine & 104 (8.3) & 105 & $31(29.5)$ & $52(49.5)$ & $12(11.4)$ & $10(9.5)$ \\
\hline Wrist & $71(5.7)$ & 71 & $3(4.2)$ & $53(74.6)$ & $8(11.3)$ & $7(9.9)$ \\
\hline Other & $92(7.3)$ & 97 & $9(9.3)$ & $63(64.9)$ & $11(11.3)$ & $14(14.4)$ \\
\hline Subjects with fracture & 274 (21.8) & 312 & $49(15.3)$ & 198 (61.9) & $37(11.6)$ & 36 (11.2) \\
\hline
\end{tabular}

Values are presented as number (\%).

Table 4. Fracture site and treatment method $(\mathrm{N}=1,255)$

\begin{tabular}{|c|c|c|c|c|c|c|}
\hline \multirow{2}{*}{ Fracture site } & \multirow{2}{*}{ Total } & \multirow{2}{*}{ No. of occurrences } & \multicolumn{4}{|c|}{ Treatment method (duplicate response) } \\
\hline & & & Surgery & Medication & Rest & Etc. (no response) \\
\hline Hip & $27(2.2)$ & 27 & $22(81.5)$ & $3(11.1)$ & 0 & $2(7.4)$ \\
\hline Knee & $12(1.0)$ & 12 & $2(16.7)$ & $8(66.7)$ & $2(16.7)$ & $1(8.3)$ \\
\hline Spine & $104(8.3)$ & 105 & $31(29.5)$ & $51(48.6)$ & $38(36.2)$ & $5(4.8)$ \\
\hline Wrist & $71(5.7)$ & 71 & $24(33.8)$ & $38(53.5)$ & $12(16.9)$ & $5(7.0)$ \\
\hline Other & $92(7.3)$ & 97 & $28(28.9)$ & $44(45.4)$ & $33(34.0)$ & $8(8.2)$ \\
\hline Subjects with fracture & $274(21.8)$ & 312 & $107(34.3)$ & $144(46.2)$ & 85 (27.2) & $21(6.7)$ \\
\hline
\end{tabular}

Values are presented as number (\%).

Table 5. Fractures before and after menopause

\begin{tabular}{|c|c|c|c|c|}
\hline Fracture site (by survey) & $\begin{array}{c}\text { No. of } \\
\text { patients }\end{array}$ & $\begin{array}{c}\text { Before } \\
\text { menopause }\end{array}$ & $\begin{array}{c}\text { After } \\
\text { menopause }\end{array}$ & $\begin{array}{l}\text { Do not } \\
\text { know }^{\text {a) }}\end{array}$ \\
\hline Spine fracture & 104 & 5 & 72 & 27 \\
\hline Hip fracture & 27 & 1 & 18 & 8 \\
\hline Wrist fracture & 71 & 8 & 51 & 12 \\
\hline Knee fracture & 12 & 1 & 5 & 6 \\
\hline All fractures including spine, femur, wrist, knee, and other fracture sites ${ }^{\mathrm{b})}$ & $274^{c /}$ & 31 & 186 & 57 \\
\hline
\end{tabular}

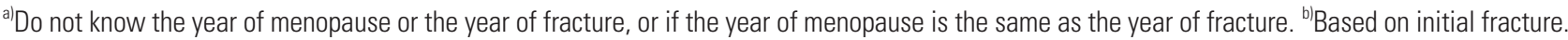
${ }^{c)}$ Among the 1,255 patients, 274 (21.8\%) responded to the survey that they had fractures. 


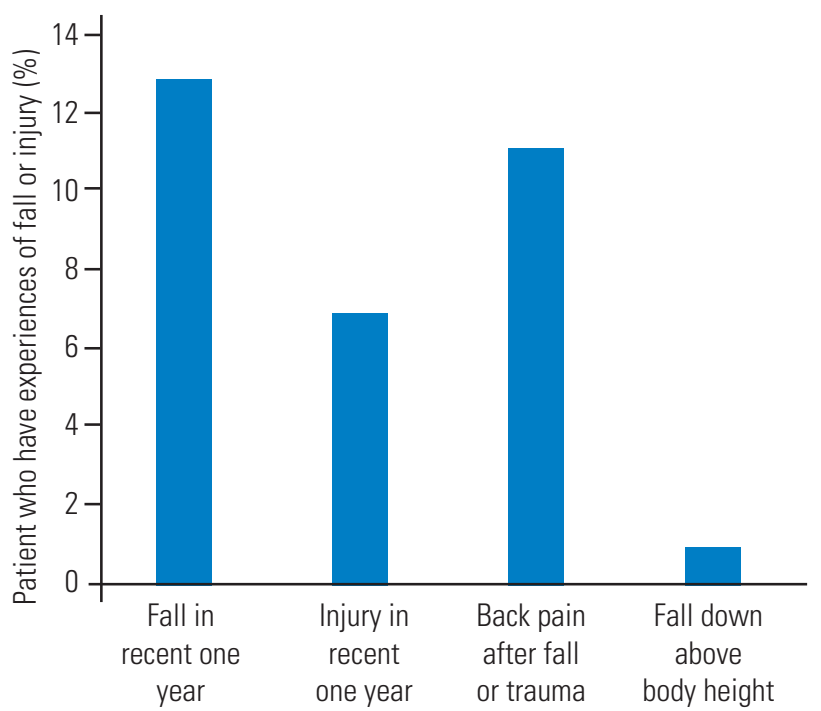

Fig. 1. History of falls in postmenopausal women who visited orthopedic outpatient clinics.

in $33.8 \%, 29.5 \%$, and $16.7 \%$ of cases, respectively. In total surgical treatment was provided in $34.3 \%$ of cases (Table 4 ).

Answers to the questionnaire indicated that of the total number of fractures $(n=277), 186$ of these occurred after menopause and only 31 fractures occurred before menopause. Fifty-seven patients did not remember whether their fracture occurred before or after the menopause (Table 5).

Among the subjects, 161 (12.8\%) had fallen within the past year and the average number of falls was 1.4 per year. A total of $6.9 \%$ of patients had experienced a trauma or traffic accident within the past year and the average number of injuries was 1.0 per year. Of these patients, 138 (11.0\%) experienced back pain following the fall or trauma. While most falls were from that was below body height, some patients $(0.9 \%)$ had experienced a fall from a distance greater than body height (Fig. 1).

\section{Factors associated with bone mineral density}

In this study, we investigated whether a family history of osteoporosis, the presence of diseases that limit exercise and walking, and previous steroid treatment were associated with the BMD of the lumbar spine, femur, and total hip. Of these criteria, the only association identified was between family history of osteoporosis and lower BMD of the lumbar spine ( $p=0.0437$ ). However, we could not confirm any association between the presence of diseases that limit exercise and walking and previous steroid treatment with a family history of osteoporosis.

\section{Discussion}

Postmenopausal women appear to have increased incidences of various comorbidities as well as decreased $\mathrm{BMD}$. The prevalence of metabolic syndrome, found in $13 \%-45 \%$ of women of reproductive age, increases by $32 \%-70 \%$ after menopause $[4,5]$. Additionally, menopause itself has been reported to be an independent risk factor for type 2 diabetes regardless of body weight and age [6,7]. In addition, the prevalence of hypertension in postmenopausal women is $41 \%$, which is higher than that in men [8]. Therefore, it is important to understand potential comorbidities found in postmenopausal fracture patients in orthopedic outpatient clinics; to date, there has been little research on this population [9]. In our study, 64.7\% of postmenopausal women who visited orthopedic outpatient clinics had concomitant diseases and more than half had chronic diseases. The incidence of these comorbid diseases is expected to increase gradually as the population ages. Patients who have parathyroid hormone disease, undergo long-term steroid usage, and exhibit rheumatoid arthritis also accounted for some of the fracture clinic outpatients. The presence of osteoporotic fractures is of particular importance in this group of patients.

Osteoporotic fractures are a major complication in postmenopausal women and are one of the major causes of socioeconomic burden [10]. Most osteoporotic fractures occur after menopause and in this study at least $67.8 \%$ (up to $88.6 \%$ ) of these fractures happened after menopause. The most common cause of osteoporotic fracture was falling, followed by minor trauma, and daily living. In this study, $12.8 \%$ of patients had experienced a fall within the year and $6.9 \%$ had experienced a trauma or traffic accident within the year. The causes of falls or trauma in elderly patients have been widely reported. In general, about half of these falls are due to jamming of the feet or slipping. Another $20 \%$ are caused by syncope, $20 \%$ are due to loss of balance, and the rest are the results of other factors [11]. When considering the risk of osteoporotic fracture, any history of falling and minor traumas should be taken into account in the outpatient setting.

The most frequent location for an osteoporotic fracture in postmenopausal women was the vertebrae. However, surgical treatment was performed in only $29 \%$ of patients with this type of fracture compared with $81.5 \%$ patients 
with hip fractures that were surgically treated. Kung [12] have stated that the surgical treatment of vertebral fractures is low because many patients are asymptomatic and the cause of the back pain is difficult to distinguish. Nonetheless, osteoporotic vertebral fractures should not be ignored considering that the presence of existing osteoporotic fractures in itself is a risk factor for another vertebral fracture, decreasing quality of life [13], and increasing mortality [14].

Several cohort studies have been conducted on the family history of osteoporosis and osteoporotic fractures. Fox et al. [15] reported that the risk of hip and wrist fracture increased by $50 \%$ and $64 \%$, respectively, in the presence of parental history of osteoporotic fracture of the hip or wrist. A family history seems to increase the risk of fracture independently of BMD [15]. Other researchers found a negative correlation between a fracture history in a sister and 10-year fracture-free survival in perimenopausal women [16]. In our study, the family history of osteoporosis and fractures were reported as $23.1 \%$ and $16.5 \%$, respectively. Few respondents knew of their family history of osteoporosis. The number of people who knew this information was lower than the number who knew about the prevalence of osteoporosis in postmenopausal women. This was likely to be underestimated in view of the low response rate.

In this study, lumbar BMD was found to be statistically lower in patients with a family history of osteoporosis than in those with no family history of the condition. According to the present study, a family history of osteoporosis may be helpful in predicting the occurrence of osteoporosis in the lumbar spine for orthopedic patients. Soroko et al. [17] reported that osteopenia occurred in more by $51 \%$ of women with a family history of osteoporosis in their cohort study of Caucasian women aged 60 to 89 years. However, there was no difference in BMD for patients with diseases that limit exercise or gait and those who had been treated with steroids for 3 months or more, which are known risk factors for osteoporosis. These results might have been limited by a failure to control other confounding factors in this study.

This study has limitations as a cross-sectional study and it is impossible to explain the causal relationship. Postmenopausal women who did not attend the outpatient clinic because of disability or other reasons could have fallen out of the study and therefore there may be a selection bias. Additionally, the collection of data on the family history of osteoporosis was memory-dependent and selfreported. Furthermore, the paternal family history was not used in this study. Nevertheless, this is the first study in which a nationwide, cross-sectional study on orthopedic outpatients was performed to examine the multifaceted approach to managing the risk factors for osteoporotic fracture in Korean postmenopausal women.

\section{Conclusions}

In conclusion, postmenopausal women visiting orthopedic outpatient clinics are a characteristic population group with a high degree of comorbidity including osteoporosis and high risk of osteoporotic fracture. Among the osteoporotic fractures exhibited by postmenopausal women, vertebral fractures may be the most frequently found, but also the most overlooked. Diagnosis of osteoporotic vertebral fractures and the evaluation of osteoporosis should not be missed for this population. A family history of osteoporosis can also be used to evaluate the risks of osteoporosis and osteoporotic fracture.

\section{Conflict of Interest}

No potential conflict of interest relevant to this article was reported.

\section{References}

1. Kontis V, Bennett JE, Mathers CD, Li G, Foreman K, Ezzati M. Future life expectancy in 35 industrialised countries: projections with a Bayesian model ensemble. Lancet 2017;389:1323-35.

2. Lee JH, Lee YH, Moon SH; TOP Study Group. Association between bone mineral density and clinical consequences: cross-sectional study of Korean postmenopausal women in an orthopaedic outpatient clinic. J Korean Med Sci 2014;29:1152-60.

3. Lee JH, Lee YH, Moon SH, Lee YS; Prevalence of Osteoporotic Vertebral Compression Fractures in Korean Post Menopausal Women Study Group. Influence of insurance benefit criteria on the administration rate of osteoporosis drugs in postmenopausal females. Clin Orthop Surg 2014;6:56-61.

4. Stefanska A, Bergmann K, Sypniewska G. Metabolic syndrome and menopause: pathophysiology, clinical and diagnostic significance. Adv Clin Chem 
2015;72:1-75.

5. Janssen I, Powell LH, Crawford S, Lasley B, SuttonTyrrell K. Menopause and the metabolic syndrome: the Study of Women's Health Across the Nation. Arch Intern Med 2008;168:1568-75.

6. Park SK, Harlow SD, Zheng H, et al. Association between changes in oestradiol and follicle-stimulating hormone levels during the menopausal transition and risk of diabetes. Diabet Med 2017;34:531-8.

7. Brand JS, van der Schouw YT, Onland-Moret NC, et al. Age at menopause, reproductive life span, and type 2 diabetes risk: results from the EPIC-InterAct study. Diabetes Care 2013;36:1012-9.

8. National Center for Health Statistics. Health, United States, 2010: with special feature on death and dying [Internet]. Hyattsville (MD): Department of Health and Human Services, Centers for Disease Control and Prevention, National Center for Health Statistics; 2011 [cited 2018 Nov 1]. Available from: https:// www.cdc.gov/nchs/data/hus/hus10.pdf.

9. Boissonnault WG. Prevalence of comorbid conditions, surgeries, and medication use in a physical therapy outpatient population: a multicentered study. J Orthop Sports Phys Ther 1999;29:506-19.

10. Singer A, Exuzides A, Spangler L, et al. Burden of illness for osteoporotic fractures compared with other serious diseases among postmenopausal women in the United States. Mayo Clin Proc 2015;90:53-62.

11. Dias JJ. An analysis of the nature of injury in fractures of the neck of the femur. Age Ageing 1987;16:373-7.

12. Kung AW. Epidemiology and diagnostic approaches to vertebral fractures in Asia. J Bone Miner Metab 2004;22:170-5.

13. Jung HJ, Park YS, Seo HY, et al. Quality of life in patients with osteoporotic vertebral compression fractures. J Bone Metab 2017;24:187-96.

14. Lau E, Ong K, Kurtz S, Schmier J, Edidin A. Mortality following the diagnosis of a vertebral compression fracture in the Medicare population. J Bone Joint Surg Am 2008;90:1479-86.

15. Fox KM, Cummings SR, Powell-Threets K, Stone K. Family history and risk of osteoporotic fracture: study of Osteoporotic Fractures Research Group. Osteoporos Int 1998;8:557-62.

16. Sirola J, Salovaara K, Tuppurainen M, Jurvelin JS, Alhava E, Kroger H. Sister's fracture history may be associated with perimenopausal bone fragility and modifies the predictability of fracture risk. Osteoporos Int 2009;20:557-65.

17. Soroko SB, Barrett-Connor E, Edelstein SL, Kritz-Silverstein D. Family history of osteoporosis and bone mineral density at the axial skeleton: the Rancho Bernardo Study. J Bone Miner Res 1994;9:761-9. 